\title{
An Eruptive Complex Solar Flare and Events in its Aftermath
}

\author{
M.L. Luoni ${ }^{1}$, C. Francile ${ }^{2}$, C. H. Mandrini ${ }^{1,3}$ and H. Cremades ${ }^{4,5}$ \\ ${ }^{1}$ Instituto de Astronomía y Física del Espacio (IAFE, UBA-CONICET), Buenos Aires, \\ Argentina \\ email: mluoni@iafe.uba.ar \\ ${ }^{2}$ Observatorio Astronómico Félix Aguilar (UNSJ), San Juan, Argentina \\ ${ }^{3}$ Facultad de Ciencias Exactas y Naturales (FCEN, UBA), Buenos Aires, Argentina \\ ${ }^{4}$ Universidad Tecnológica Nacional - Facultad Regional Mendoza, Mendoza, Argentina \\ ${ }^{5}$ Consejo Nacional de Investigaciones Científicas y Técnicas (CONICET), Mendoza, Argentina
}

\begin{abstract}
We present a study of the M6.6 flare that occurred on 13 February 2011 in AR 11158. The flare was accompanied by a CME and EUV waves. We use multiwavelength observations from the ground: $\mathrm{H}$-alpha Solar Telescope for Argentina (HASTA), and space: Helioseismic and Magnetic Imager (HMI) and Atmospheric Imaging Assembly (AIA), both onboard the Solar and Dynamic Observatory (SDO).
\end{abstract}

Keywords. Sun: flares, Sun: corona, Sun: coronal mass ejections (CMEs), Sun: EUV waves.

\section{Introduction}

Impulsive releases of large amounts of energy in flares are frequently accompanied by CME disturbances and wave-like features propagating in the solar corona. The physical processes that underlie these events are still unclear due to the complex mechanisms involved. In general, the coronal propagating disturbances that follow a CME cannot be properly identified due to the line-of-sight overlap of different features, such as real MHD waves or those derived from the magnetic coronal reconfiguration. With the aim of contributing to the understanding of some of these aspects, we analyze the M6.6 flare in AR 11158, which occured on 13 February 2011, associated with a coronal mass ejection (CME) and EUV waves. We use ground-based observations from the H-alpha Solar Telescope for Argentina (HASTA), and space observations from the Helioseismic and Magnetic Imager (HMI) and the Atmospheric Imaging Assembly (AIA), onboard the Solar Dynamics Observatory (SDO).

\section{Observations and data analysis}

On 13 February 2011 an M6.6 flare was observed in AR 11158. This event was seen in the chromosphere with HASTA (Bagalá et al. 1999, Francile et al. 2008). In Figure 1 we compute the derivative of the $\mathrm{H} \alpha$ light curve and determine the flare onset time as 17:32:33 UT (Fig. 1). The AR remained activated during one hour and a second impulsive peak was observed at 18:25 UT.

Fig. 2 shows the evolution of AR 11158 from 10 to 13 February 2011 as observed with HMI/SDO at 17:01:30 UT on each day. We identify two small bipoles that approached as they emerged. On 13 February the AR displayed a quadrupolar magnetic field configuration. We infer that the observed flux emergence, accompanied by shearing motions, led to the interaction of the structures anchored to the approaching bipoles with the consequent energy release that originated the M6.6 flare and CME ejection. 


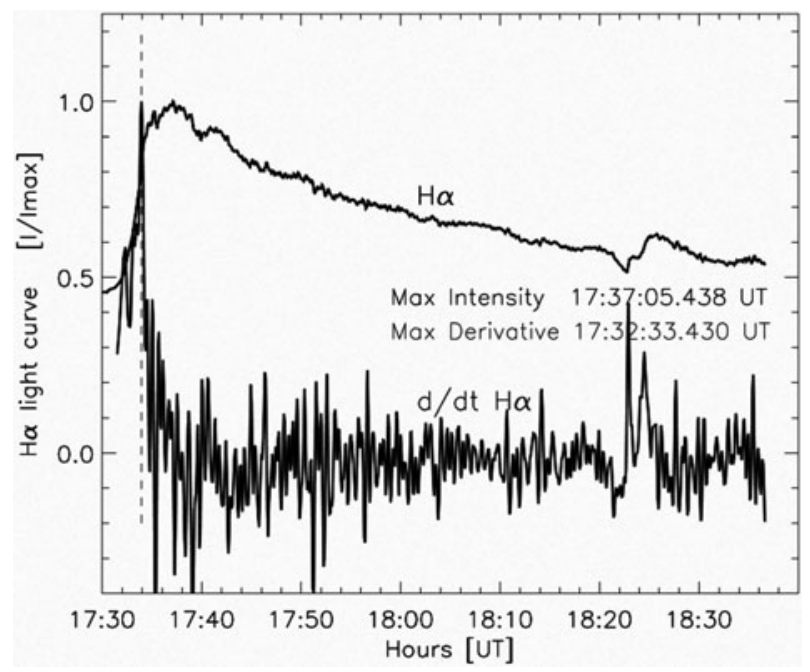

Figure 1. Flare light curve obtained from HASTA.
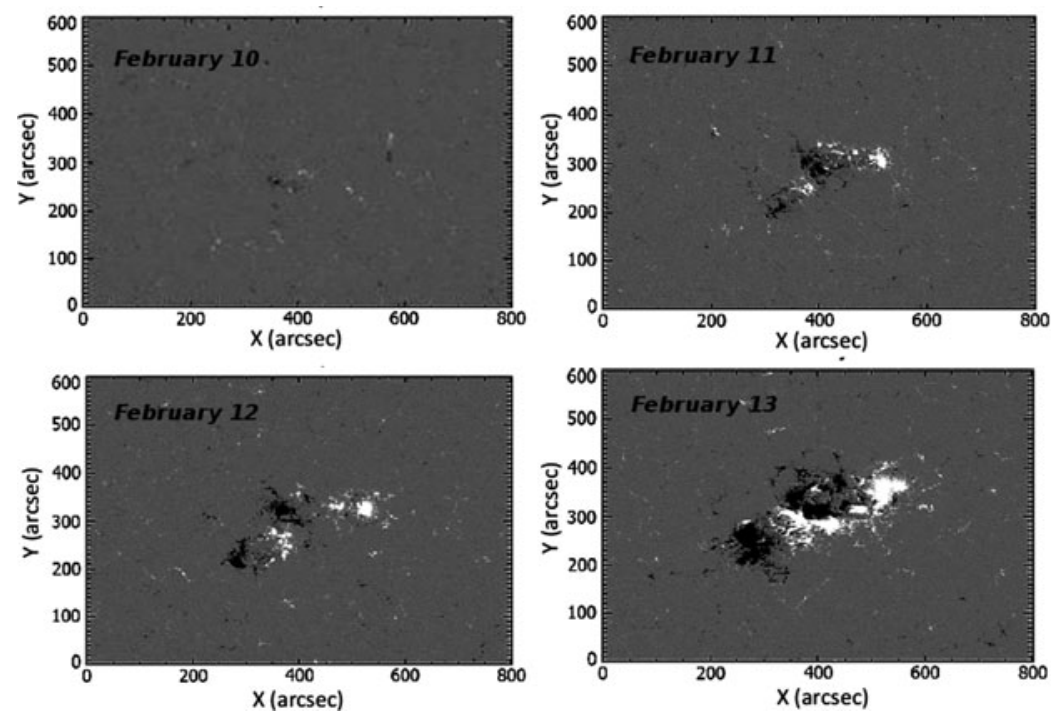

Figure 2. Magnetic evolution of AR 11158. The images correspond to HMI/SDO taken at 17:01:30 UT on each day.

\subsection{EUV waves}

After the flare initiation and CME disruption, coronal EUV waves were visible. We obtained running-difference images of AIA images in the $193 \AA$ band. To analyze the temporal and spatial evolution of the waves we applied the intensity profile technique discussed by Vršnak et al. (2002) to these images. The intensity profiles were computed along angular sectors traced every $5^{\circ}$ departing from the point where the flare intensity was strongest (S20E04), which we call the radiant point (RP). Seventy two sectors were traced and numbered beginning from that centered at the solar South Pole in a counterclockwise direction from 0 to 71 .

To investigate the evolution of the wave event in different angular sectors, we apply a stack plot procedure as done in Francile et al. (2016). Distance-time (DT) maps are 


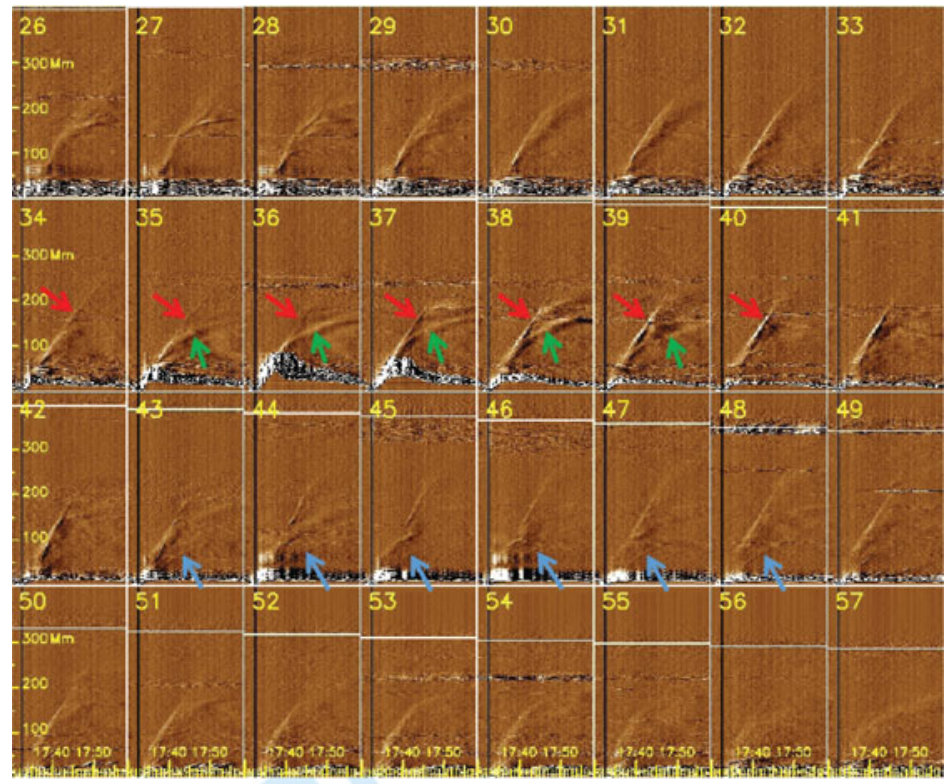

Figure 3. Stack plots, sectors 26 to 57 . The red, green, and blue arrows indicate different EUV propagating perturbations.

shown for sectors 26 to 57 in Fig. 3. The abscissae are in seconds while the ordinates are the plane of sky distance measured from the RP.

The first appearance of a EUV disturbance is observed as a sharp, well-defined, and slowly decelerating line between sectors 29 to 51, denoted with red arrows in some of the central panels. This disturbance resembles an S-type EIT wave or a shock wavefront, which originated at the flare and CME onset. This disturbance can also be driven by the CME bubble as discussed by Patsourakos et al. (2012). The green arrows in Fig. 3 denote a second propagating disturbance, which is more blurred and exhibit a higher decelerating path, detaching from the sharp front at $\approx 100 \mathrm{Mm}$ from the RP. The blue arrows indicate a thin and sharp third EUV disturbance not connected to the others.

\subsection{Kinematics}

We estimate the kinematic parameters of the first appearing sharp wavefront (pointed by red arrows in Fig. 3) by fitting linear and quadratic curves for every sector . For this purpose, we draw points over the fronts using a visual method and fit curves to these points for sectors 26 to 51 for the time interval 17:32:33 to 17:37:33 UT.

A nearly homogeneous speed, with a mean of $446.7 \mathrm{~km} \mathrm{~s}^{-1}$, is derived from the linear fittings. A predominance of negative accelerations, with a mean of $-0.112 \mathrm{~km} \mathrm{~s}^{-2}$, results from the quadratic fittings. We also calculate the speeds extrapolated to the flare onset time (17:32:33 UT) and 5 min later. These values show high dispersion, with a mean of $512 \mathrm{~km} \mathrm{~s}^{-1}$ at the flare onset and a decay to approximately $478 \mathrm{~km} \mathrm{~s}^{-1} 5$ minutes later.

\section{3. $C M E$}

The CME was observed by the Large Angle and Spectroscopic Coronagraph (LASCO) onboard the Solar and Heliospheric Observatory (SOHO). According to the CDAW SOHO/LASCO CME Catalog, the event associated with the EUV wave was a poor partial halo, first detected in running difference images at 18:36 UT. The height-time points measured as projected in the plane of the sky are shown in Fig. 4. A linear fit 

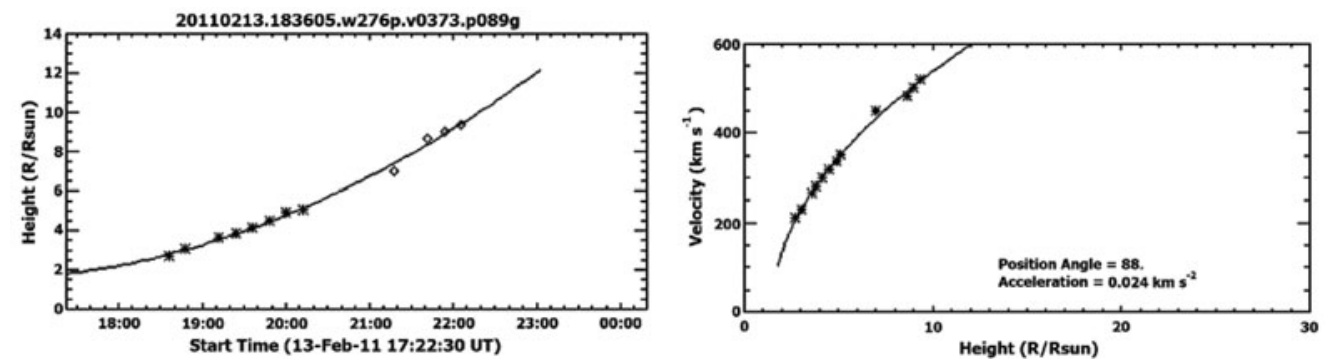

Figure 4. Left: Quadratic fit to the height-time points. Right: Velocity of the CME, both measured projected in the plane of the sky.

indicates a mean speed of $373 \mathrm{~km} \mathrm{~s}^{-1}$. The measured points can also be fitted to a quadratic curve, which gives as a result an acceleration of $0.024 \mathrm{~km} \mathrm{~s}^{-2}$ and implies a speed of $517 \mathrm{~km} \mathrm{~s}^{-1}$ at the height of the last measured point. These speed values do not necessarily correspond to the propagation speed of the CME, but rather to its expansion speed, given the vantage point of SOHO. From the perspective of the Solar Terrestrial Relations Observatory (STEREO), the CME appears in the field of view of COR1 at 17:45 UT. In the FOV of COR2, the CME is detected as an unstructured event, without exhibiting signatures of a three-part or flux rope structure.

\section{Discussion and Conclusions}

Our analysis by sectors of the first EUV wavefront indicates an almost homogeneous angular behavior. We determine a mean speed of $447 \mathrm{~km} \mathrm{~s}^{-1}$ and a mean acceleration of $-0.112 \mathrm{~km} \mathrm{~s}^{-2}$. This wavefront has the characteristics of an MHD fast magnetosonic shock of the blast type, i.e. a shock originated by a short lived 3D piston. This 3D piston can be associated with a pressure pulse (flare origin) or with the initial acceleration of the CME (see the discussion in Francile et al. 2016). From our analysis it is not possible to discern the origin of the EUV shock.

The green arrows in Fig. 3 denote a second and slow propagating disturbance. Probably it is not a real wave because it could be originated by the outskirt of the first EUV front what interacts with the lower and denser coronal layers close to solar surface while it expands. The third EUV disturbance (blue arrows in Fig. 3) is probably the trace of a real MHD wave since it exhibits a well defined and thin front. We measure a mean speed of $204.8 \mathrm{~km} \mathrm{~s}^{-1}$ and an acceleration of $-0.043 \mathrm{~km} \mathrm{~s}^{-2}$, which suggest that it can be of the slow magnetosonic type and, probably, in a shock regime. Fast and slow magnetosonic shock waves are compressional waves, which could explain the observed enhancements of the EUV emission. Existence of multiple EUV waves has been reported in several cases (Warmuth et al. 2015 and references therein). From the analysis of the CME kinematic evolution it is not possible to infer a clear relation with the measured EUV waves.

\section{References}

Bagalá, L. G., Bauer, O. H., Fernández Borda, R., Francile, C., Haerendel, G., \& Rieger, R. 1999, in ESA-SP 448, p.469

Francile, C., Castro, J. I., Leuzzi, L., Luoni, M. L., Rovira, M., \& Cornudella, A. 2008, Bol. Asoc. Arg. Astron., 51, 339

Francile, C., López, F., Cremades, H., Mandrini, C. H., Luoni, M. L., \& Long, D. 2016, Sol. Phys., 291, 3217

Patsourakos, S. \& Vourlidas, A. 2012, Sol. Phys., 281, 187

Vršnak, B., Warmuth, A., Brajša, R., \& Hanslmeier, A. 2002, A\&A, 394, 299

Warmuth, A. 2015 Living Reviews in Solar Physics 